\title{
Relativistic Atomic Calculations of Spectral Parameters of Be-Like Tungsten Ion - Supplement
}

\author{
K. HAMASHA \\ Department of Basic Sciences, Al-Huson University College, \\ Al-Balqa Applied University, Al-Salt 19117, Jordan \\ Received: 27.11.2020 \& Accepted: 12.01.2021 \\ Doi: 10.12693/APhysPolA.139.151.S1 \\ *e-mail: khamasha@bau.edu.jo \\ topics: electric dipole transitions, forbidden transitions, oscillator strengths, transition rates
}

TABLE SI

Transition energy $(\Delta E[\mathrm{eV}])$, wavelength $(\lambda[\AA])$, radiative transition rates $\left(A_{r}\left[\mathrm{~s}^{-1}\right]\right)$ and weighted oscillator strengths $\left(g f_{i j}\right)$ for strong electric-quadrupole transitions (E2) of Be-like tungsten ion calculated by RCI method of FAC.

\begin{tabular}{|c|c|c|c|c|c|c|c|}
\hline Upper state & $J_{\text {up }}$ & Lower state & $J_{\text {low }}$ & $\Delta E[\mathrm{eV}]$ & $g f_{i j}$ & $A_{r}\left[\mathrm{~s}^{-1}\right]$ & $\lambda[\AA]$ \\
\hline $2 s_{1 / 2} 3 s_{1 / 2}$ & 0 & $2 p_{1 / 2} 2 p_{3 / 2}$ & 2 & 9078.3 & $1.788 \times 10^{-5}$ & $6.3935 \times 10^{10}$ & 1.3659 \\
\hline $2 p_{3 / 2} 3 p_{1 / 2}$ & 2 & $2 p_{3 / 2}^{2}$ & 0 & 9285.5 & $8.232 \times 10^{-4}$ & $6.1593 \times 10^{11}$ & 1.3354 \\
\hline $2 p_{1 / 2} 3 p_{1 / 2}$ & 1 & $2 p_{1 / 2} 2 p_{3 / 2}$ & 2 & 9306.9 & $1.259 \times 10^{-3}$ & $1.5774 \times 10^{12}$ & 1.3324 \\
\hline $2 s_{1 / 2} 3 p_{1 / 2}$ & 1 & $2 s_{1 / 2} 2 p_{3 / 2}$ & 1 & 9321.8 & $1.224 \times 10^{-3}$ & $1.5380 \times 10^{12}$ & 1.3302 \\
\hline $2 p_{1 / 2} 3 p_{1 / 2}$ & 1 & $2 p_{1 / 2} 2 p_{3 / 2}$ & 1 & 9325.1 & $1.264 \times 10^{-3}$ & $1.5893 \times 10^{12}$ & 1.3297 \\
\hline $2 p_{3 / 2} 3 p_{1 / 2}$ & 1 & $2 p_{3 / 2}^{2}$ & 2 & 9353.0 & $1.294 \times 10^{-3}$ & $1.6373 \times 10^{12}$ & 1.3258 \\
\hline $2 p_{3 / 2} 3 p_{1 / 2}$ & 2 & $2 p_{3 / 2}^{2}$ & 2 & 9354.5 & $2.958 \times 10^{-3}$ & $2.2461 \times 10^{12}$ & 1.3256 \\
\hline $2 p_{1 / 2} 3 p_{1 / 2}$ & 0 & $2 p_{1 / 2} 2 p_{3 / 2}$ & 2 & 9363.0 & $8.240 \times 10^{-4}$ & $3.1346 \times 10^{12}$ & 1.3244 \\
\hline $2 s_{1 / 2} 3 p_{1 / 2}$ & 1 & $2 s_{1 / 2} 2 p_{3 / 2}$ & 2 & 9412.8 & $1.308 \times 10^{-3}$ & $1.6759 \times 10^{12}$ & 1.3174 \\
\hline $2 s_{1 / 2} 3 p_{1 / 2}$ & 0 & $2 s_{1 / 2} 2 p_{3 / 2}$ & 2 & 9412.9 & $8.752 \times 10^{-4}$ & $3.3650 \times 10^{12}$ & 1.3173 \\
\hline $2 s_{1 / 2} 4 s_{1 / 2}$ & 0 & $2 p_{1 / 2} 2 p_{3 / 2}$ & 2 & 12810.3 & $1.164 \times 10^{-5}$ & $8.2901 \times 10^{10}$ & 0.9680 \\
\hline $2 p_{3 / 2} 4 p_{1 / 2}$ & 2 & $2 p_{3 / 2}^{2}$ & 0 & 12986.6 & $1.688 \times 10^{-4}$ & $2.4712 \times 10^{11}$ & 0.9548 \\
\hline $2 p_{1 / 2} 4 p_{1 / 2}$ & 1 & $2 p_{1 / 2} 2 p_{3 / 2}$ & 2 & 13016.9 & $1.713 \times 10^{-4}$ & $4.1993 \times 10^{11}$ & 0.9526 \\
\hline $2 s_{1 / 2} 4 p_{1 / 2}$ & 1 & $2 S_{1 / 2} 2 p_{3 / 2}$ & 1 & 13028.0 & $2.428 \times 10^{-4}$ & $5.9598 \times 10^{11}$ & 0.9518 \\
\hline $2 s_{1 / 2} 5 s_{1 / 2}$ & 0 & $2 p_{3 / 2}^{2}$ & 2 & 13029.9 & $9.172 \times 10^{-6}$ & $6.7568 \times 10^{10}$ & 0.9517 \\
\hline $2 s_{1 / 2} 6 s_{1 / 2}$ & 0 & $2 p_{3 / 2}^{2}$ & 2 & 13935.7 & $9.703 \times 10^{-6}$ & $8.1765 \times 10^{10}$ & 0.8898 \\
\hline $2 s_{1 / 2} 6 d_{5 / 2}$ & 2 & $2 p_{3 / 2}^{2}$ & 2 & 14016.7 & $8.361 \times 10^{-6}$ & $1.4255 \times 10^{10}$ & 0.8847 \\
\hline $2 s_{1 / 2} 4 d_{3 / 2}$ & 2 & $2 p_{1 / 2}^{2}$ & 0 & 14483.1 & $1.783 \times 10^{-5}$ & $3.2450 \times 10^{10}$ & 0.8562 \\
\hline $2 s_{1 / 2} 5 s_{1 / 2}$ & 0 & $2 p_{1 / 2} 2 p_{3 / 2}$ & 2 & 14504.6 & $1.725 \times 10^{-5}$ & $1.5750 \times 10^{11}$ & 0.8549 \\
\hline $2 s_{1 / 2} 4 d_{5 / 2}$ & 2 & $2 p_{1 / 2}^{2}$ & 0 & 14537.3 & $1.986 \times 10^{-5}$ & $3.6425 \times 10^{10}$ & 0.8530 \\
\hline $2 s_{1 / 2} 6 g_{7 / 2}$ & 4 & $2 p_{1 / 2} 2 p_{3 / 2}$ & 2 & 15500.3 & $8.890 \times 10^{-6}$ & $1.0298 \times 10^{10}$ & 0.8000 \\
\hline $2 s_{1 / 2} 6 g_{9 / 2}$ & 4 & $2 p_{1 / 2} 2 p_{3 / 2}$ & 2 & 15504.6 & $9.246 \times 10^{-6}$ & $1.0716 \times 10^{10}$ & 0.7998 \\
\hline $2 p_{3 / 2} 6 p_{1 / 2}$ & 2 & $2 p_{3 / 2}^{2}$ & 0 & 15571.3 & $4.602 \times 10^{-5}$ & $9.6830 \times 10^{10}$ & 0.7963 \\
\hline $2 p_{1 / 2} 6 p_{1 / 2}$ & 1 & $2 p_{1 / 2} 2 p_{3 / 2}$ & 2 & 15610.4 & $6.456 \times 10^{-5}$ & $2.2756 \times 10^{11}$ & 0.7943 \\
\hline $2 s_{1 / 2} 6 p_{1 / 2}$ & 1 & $2 s_{1 / 2} 2 p_{3 / 2}$ & 1 & 15614.2 & $5.584 \times 10^{-5}$ & $1.9690 \times 10^{11}$ & 0.7941 \\
\hline $2 p_{3 / 2} 5 s_{1 / 2}$ & 1 & $2 s_{1 / 2} 2 p_{1 / 2}$ & 1 & 17935.8 & $2.357 \times 10^{-6}$ & $1.0966 \times 10^{10}$ & 0.6914 \\
\hline
\end{tabular}


TABLE SII

Transition energy $(\Delta E[\mathrm{eV}])$, wavelength $(\lambda[\AA])$, radiative transition rates $\left(A_{r}\left[\mathrm{~s}^{-1}\right]\right)$ and weighted oscillator strengths $\left(g f_{i j}\right)$ for strong electric-octupole transitions (E3) of Be-like tungsten ion calculated by RCI method of FAC.

\begin{tabular}{|c|c|c|c|c|c|c|c|}
\hline Upper state & $J_{\text {up }}$ & Lower state & $J_{\text {low }}$ & $\Delta E[\mathrm{eV}]$ & $g f_{i j}$ & $A_{r}\left[\mathrm{~s}^{-1}\right]$ & $\lambda[\AA]$ \\
\hline $2 p_{3 / 2} 3 d_{3 / 2}$ & 3 & $2 p_{3 / 2}^{2}$ & 0 & 9759.5 & $5.746 \times 10^{-6}$ & $3.3924 \times 10^{9}$ & 1.2706 \\
\hline $2 p_{1 / 2} 3 d_{3 / 2}$ & 2 & $2 p_{1 / 2} 2 p_{3 / 2}$ & 2 & 9785.8 & $6.993 \times 10^{-6}$ & $5.8116 \times 10^{9}$ & 1.2671 \\
\hline $2 s_{1 / 2} 3 d_{3 / 2}$ & 2 & $2 s_{1 / 2} 2 p_{3 / 2}$ & 1 & 9798.5 & $9.169 \times 10^{-6}$ & $7.6399 \times 10^{9}$ & 1.2655 \\
\hline $2 p_{1 / 2} 3 d_{3 / 2}$ & 2 & $2 p_{1 / 2} 2 p_{3 / 2}$ & 1 & 9804.0 & $1.059 \times 10^{-5}$ & $8.8323 \times 10^{9}$ & 1.2648 \\
\hline $2 p_{1 / 2} 3 d_{3 / 2}$ & 1 & $2 p_{1 / 2} 2 p_{3 / 2}$ & 2 & 9813.2 & $1.052 \times 10^{-5}$ & $1.4649 \times 10^{10}$ & 1.2636 \\
\hline $2 p_{3 / 2} 3 d_{3 / 2}$ & 2 & $2 p_{3 / 2}^{2}$ & 2 & 9819.8 & $1.490 \times 10^{-5}$ & $1.2465 \times 10^{10}$ & 1.2628 \\
\hline $2 p_{3 / 2} 3 d_{3 / 2}$ & 3 & $2 p_{3 / 2}^{2}$ & 2 & 9828.6 & $1.751 \times 10^{-5}$ & $1.0483 \times 10^{10}$ & 1.2616 \\
\hline $2 p_{3 / 2} 3 d_{3 / 2}$ & 1 & $2 p_{3 / 2}^{2}$ & 2 & 9832.9 & $3.849 \times 10^{-6}$ & $5.3833 \times 10^{9}$ & 1.2611 \\
\hline $2 s_{1 / 2} 3 d_{3 / 2}$ & 1 & $2 s_{1 / 2} 2 p_{3 / 2}$ & 2 & 9884.2 & $1.076 \times 10^{-5}$ & $1.5210 \times 10^{10}$ & 1.2545 \\
\hline $2 s_{1 / 2} 3 d_{3 / 2}$ & 2 & $2 s_{1 / 2} 2 p_{3 / 2}$ & 2 & 9889.6 & $8.352 \times 10^{-6}$ & $7.0893 \times 10^{9}$ & 1.2538 \\
\hline $2 s_{1 / 2} 4 p_{1 / 2}$ & 1 & $2 p_{1 / 2} 2 p_{3 / 2}$ & 2 & 12830.7 & $1.150 \times 10^{-7}$ & $2.7376 \times 10^{8}$ & 0.9664 \\
\hline $2 p_{1 / 2} 4 s_{1 / 2}$ & 1 & $2 p_{1 / 2} 2 p_{3 / 2}$ & 2 & 12995.5 & $7.312 \times 10^{-8}$ & $1.7861 \times 10^{8}$ & 0.9542 \\
\hline $2 s_{1 / 2} 4 p_{3 / 2}$ & 1 & $2 p_{1 / 2} 2 p_{3 / 2}$ & 2 & 13020.8 & $1.953 \times 10^{-7}$ & $4.7902 \times 10^{8}$ & 0.9523 \\
\hline $2 s_{1 / 2} 5 p_{1 / 2}$ & 1 & $2 p_{3 / 2}^{2}$ & 2 & 13040.3 & $4.416 \times 10^{-7}$ & $1.0861 \times 10^{9}$ & 0.9509 \\
\hline $2 s_{1 / 2} 4 f_{7 / 2}$ & 3 & $2 p_{1 / 2} 2 p_{3 / 2}$ & 2 & 13111.7 & $1.164 \times 10^{-7}$ & $1.2399 \times 10^{8}$ & 0.9457 \\
\hline $2 s_{1 / 2} 6 p_{1 / 2}$ & 1 & $2 p_{3 / 2}^{2}$ & 2 & 13942.2 & $1.406 \times 10^{-6}$ & $3.9533 \times 10^{9}$ & 0.8894 \\
\hline $2 s_{1 / 2} 6 f_{5 / 2}$ & 3 & $2 p_{3 / 2}^{2}$ & 0 & 13948.8 & $3.216 \times 10^{-7}$ & $3.8791 \times 10^{8}$ & 0.8890 \\
\hline $2 s_{1 / 2} 6 f_{7 / 2}$ & 3 & $2 p_{3 / 2}^{2}$ & 0 & 13956.2 & $4.883 \times 10^{-7}$ & $5.8952 \times 10^{8}$ & 0.8885 \\
\hline $2 s_{1 / 2} 6 p_{3 / 2}$ & 1 & $2 p_{3 / 2}^{2}$ & 2 & 13996.4 & $2.747 \times 10^{-6}$ & $7.7840 \times 10^{9}$ & 0.8859 \\
\hline $2 s_{1 / 2} 6 f_{5 / 2}$ & 3 & $2 p_{3 / 2}^{2}$ & 2 & 14018.0 & $1.418 \times 10^{-6}$ & $1.7277 \times 10^{9}$ & 0.8846 \\
\hline $2 s_{1 / 2} 6 f_{7 / 2}$ & 3 & $2 p_{1 / 2} 2 p_{3 / 2}$ & 2 & 15500.2 & $3.981 \times 10^{-6}$ & $5.9285 \times 10^{9}$ & 0.8000 \\
\hline $2 s_{1 / 2} 6 h_{9 / 2}$ & 5 & $2 p_{1 / 2} 2 p_{3 / 2}$ & 2 & 15504.6 & $1.265 \times 10^{-6}$ & $1.1996 \times 10^{9}$ & 0.7998 \\
\hline $2 s_{1 / 2} 6 h_{11 / 2}$ & 5 & $2 p_{1 / 2} 2 p_{3 / 2}$ & 2 & 15507.4 & $1.287 \times 10^{-6}$ & $1.2209 \times 10^{9}$ & 0.7996 \\
\hline $2 p_{1 / 2} 6 s_{1 / 2}$ & 1 & $2 p_{1 / 2} 2 p_{3 / 2}$ & 2 & 15603.9 & $9.069 \times 10^{-8}$ & $3.1940 \times 10^{8}$ & 0.7947 \\
\hline $2 p_{3 / 2} 6 d_{3 / 2}$ & 3 & $2 p_{3 / 2}^{2}$ & 0 & 15630.1 & $1.128 \times 10^{-6}$ & $1.7081 \times 10^{9}$ & 0.7933 \\
\hline $2 p_{3 / 2} 5 d_{3 / 2}$ & 3 & $2 p_{1 / 2}^{2}$ & 0 & 17768.4 & $6.328 \times 10^{-8}$ & $1.2384 \times 10^{8}$ & 0.6979 \\
\hline $2 p_{3 / 2} 5 d_{5 / 2}$ & 3 & $2 p_{1 / 2}^{2}$ & 0 & 17796.0 & $5.570 \times 10^{-8}$ & $1.0935 \times 10^{8}$ & 0.6968 \\
\hline $2 p_{3 / 2} 5 p_{1 / 2}$ & 2 & $2 s_{1 / 2} 2 p_{1 / 2}$ & 1 & 17948.8 & $2.333 \times 10^{-7}$ & $6.5221 \times 10^{8}$ & 0.6909 \\
\hline $2 p_{3 / 2} 5 p_{3 / 2}$ & 3 & $2 s_{1 / 2} 2 p_{1 / 2}$ & 1 & 18039.2 & $3.110 \times 10^{-7}$ & $6.2733 \times 10^{8}$ & 0.6874 \\
\hline $2 p_{3 / 2} 5 p_{3 / 2}$ & 2 & $2 s_{1 / 2} 2 p_{1 / 2}$ & 1 & 18043.7 & $5.946 \times 10^{-7}$ & $1.6800 \times 10^{9}$ & 0.6872 \\
\hline
\end{tabular}


TABLE SIII

Transition energy $(\Delta E[\mathrm{eV}])$, wavelength $(\lambda[\AA])$, radiative transition rates $\left(A_{r}\left[\mathrm{~s}^{-1}\right]\right)$ and weighted oscillator strengths $\left(g f_{i j}\right)$ for strong magnetic-dipole transitions (M1) of Be-like tungsten ion calculated by RCI method of FAC.

\begin{tabular}{|c|c|c|c|c|c|c|c|}
\hline Upper state & $J_{\text {up }}$ & Lower state & $J_{\text {low }}$ & $\Delta E[\mathrm{eV}]$ & $g f_{i j}$ & $A_{r}\left[\mathrm{~s}^{-1}\right]$ & $\lambda[\AA]$ \\
\hline $2 s_{1 / 2} 3 s_{1 / 2}$ & 0 & $2 p_{1 / 2} 2 p_{3 / 2}$ & 1 & 9096.5 & $1.638 \times 10^{-7}$ & $5.8810 \times 10^{8}$ & 1.3632 \\
\hline $2 p_{3 / 2} 3 p_{1 / 2}$ & 1 & $2 p_{3 / 2}^{2}$ & 0 & 9284.0 & $1.579 \times 10^{-5}$ & $1.9680 \times 10^{10}$ & 1.3356 \\
\hline $2 p_{1 / 2} 3 p_{1 / 2}$ & 1 & $2 p_{1 / 2} 2 p_{3 / 2}$ & 2 & 9306.9 & $4.065 \times 10^{-5}$ & $5.0933 \times 10^{10}$ & 1.3324 \\
\hline $2 s_{1 / 2} 3 p_{1 / 2}$ & 1 & $2 s_{1 / 2} 2 p_{3 / 2}$ & 1 & 9321.8 & $7.820 \times 10^{-6}$ & $9.8285 \times 10^{9}$ & 1.3302 \\
\hline $2 s_{1 / 2} 3 p_{1 / 2}$ & 0 & $2 s_{1 / 2} 2 p_{3 / 2}$ & 1 & 9321.9 & $1.641 \times 10^{-5}$ & $6.1892 \times 10^{10}$ & 1.3302 \\
\hline $2 p_{1 / 2} 3 p_{1 / 2}$ & 1 & $2 p_{1 / 2} 2 p_{3 / 2}$ & 1 & 9325.1 & $8.116 \times 10^{-6}$ & $1.0208 \times 10^{10}$ & 1.3297 \\
\hline $2 p_{3 / 2} 3 p_{1 / 2}$ & 1 & $2 p_{3 / 2}^{2}$ & 2 & 9353.0 & $4.086 \times 10^{-5}$ & $5.1695 \times 10^{10}$ & 1.3258 \\
\hline $2 p_{3 / 2} 3 p_{1 / 2}$ & 2 & $2 p_{3 / 2}^{2}$ & 2 & 9354.5 & $4.077 \times 10^{-5}$ & $3.0963 \times 10^{10}$ & 1.3256 \\
\hline $2 p_{1 / 2} 3 p_{1 / 2}$ & 0 & $2 p_{1 / 2} 2 p_{3 / 2}$ & 1 & 9381.2 & $1.549 \times 10^{-5}$ & $5.9142 \times 10^{10}$ & 1.3218 \\
\hline $2 s_{1 / 2} 3 p_{1 / 2}$ & 1 & $2 s_{1 / 2} 2 p_{3 / 2}$ & 2 & 9412.8 & $4.014 \times 10^{-5}$ & $5.1445 \times 10^{10}$ & 1.3174 \\
\hline $2 p_{3 / 2} 4 p_{1 / 2}$ & 1 & $2 p_{3 / 2}^{2}$ & 0 & 12986.3 & $4.624 \times 10^{-6}$ & $1.1279 \times 10^{10}$ & 0.9549 \\
\hline $2 p_{1 / 2} 4 p_{1 / 2}$ & 1 & $2 p_{1 / 2} 2 p_{3 / 2}$ & 2 & 13016.9 & $7.895 \times 10^{-6}$ & $1.9348 \times 10^{10}$ & 0.9526 \\
\hline $2 s_{1 / 2} 4 p_{1 / 2}$ & 0 & $2 s_{1 / 2} 2 p_{3 / 2}$ & 1 & 13027.8 & $4.833 \times 10^{-6}$ & $3.5590 \times 10^{10}$ & 0.9518 \\
\hline $2 s_{1 / 2} 4 p_{1 / 2}$ & 1 & $2 s_{1 / 2} 2 p_{3 / 2}$ & 1 & 13028.0 & $2.311 \times 10^{-6}$ & $5.6745 \times 10^{9}$ & 0.9518 \\
\hline $2 s_{1 / 2} 4 d_{3 / 2}$ & 1 & $2 p_{1 / 2} 2 p_{3 / 2}$ & 2 & 13031.6 & $4.007 \times 10^{-6}$ & $9.8412 \times 10^{9}$ & 0.9515 \\
\hline $2 s_{1 / 2} 4 s_{1 / 2}$ & 1 & $2 p_{1 / 2}^{2}$ & 0 & 14253.0 & $5.004 \times 10^{-8}$ & $1.4704 \times 10^{8}$ & 0.8700 \\
\hline $2 p_{1 / 2} 4 p_{1 / 2}$ & 1 & $2 p_{1 / 2}^{2}$ & 0 & 14471.2 & $3.659 \times 10^{-7}$ & $1.1082 \times 10^{9}$ & 0.8569 \\
\hline $2 s_{1 / 2} 4 d_{3 / 2}$ & 1 & $2 p_{1 / 2}^{2}$ & 0 & 14485.9 & $1.576 \times 10^{-7}$ & $4.7841 \times 10^{8}$ & 0.8560 \\
\hline $2 p_{3 / 2} 4 p_{1 / 2}$ & 1 & $2 p_{1 / 2} 2 p_{3 / 2}$ & 2 & 14530.3 & $6.093 \times 10^{-7}$ & $1.8607 \times 10^{9}$ & 0.8534 \\
\hline $2 p_{3 / 2} 4 p_{1 / 2}$ & 2 & $2 p_{1 / 2} 2 p_{3 / 2}$ & 2 & 14530.7 & $7.329 \times 10^{-7}$ & $1.3430 \times 10^{9}$ & 0.8534 \\
\hline $2 p_{3 / 2} 6 p_{1 / 2}$ & 1 & $2 p_{3 / 2}^{2}$ & 0 & 15571.0 & $1.047 \times 10^{-6}$ & $3.6726 \times 10^{9}$ & 0.7964 \\
\hline $2 p_{1 / 2} 6 p_{1 / 2}$ & 1 & $2 p_{1 / 2} 2 p_{3 / 2}$ & 2 & 15610.4 & $2.707 \times 10^{-6}$ & $9.5405 \times 10^{9}$ & 0.7943 \\
\hline $2 s_{1 / 2} 6 p_{1 / 2}$ & 0 & $2 s_{1 / 2} 2 p_{3 / 2}$ & 1 & 15613.8 & $1.124 \times 10^{-6}$ & $1.1894 \times 10^{10}$ & 0.7942 \\
\hline $2 s_{1 / 2} 6 p_{1 / 2}$ & 1 & $2 s_{1 / 2} 2 p_{3 / 2}$ & 1 & 15614.2 & $5.431 \times 10^{-7}$ & $1.9151 \times 10^{9}$ & 0.7941 \\
\hline $2 p_{3 / 2} 6 p_{3 / 2}$ & 1 & $2 p_{3 / 2}^{2}$ & 0 & 15623.9 & $5.814 \times 10^{-7}$ & $2.0529 \times 10^{9}$ & 0.7937 \\
\hline
\end{tabular}


TABLE SIV

Transition energy $(\Delta E[\mathrm{eV}])$, wavelength $(\lambda[\AA])$, radiative transition rates $\left(A_{r}\left[\mathrm{~s}^{-1}\right]\right)$ and weighted oscillator strengths $\left(g f_{i j}\right)$ for strong magnetic-quadrupole transitions (M2) of Be-like tungsten ion calculated by RCI method of FAC.

\begin{tabular}{|c|c|c|c|c|c|c|c|}
\hline Upper state & $J_{\text {up }}$ & Lower state & $J_{\text {low }}$ & $\Delta \mathrm{E}[\mathrm{eV}]$ & $g f_{i j}$ & $A_{r}\left[\mathrm{~s}^{-1}\right]$ & $\lambda[\AA]$ \\
\hline $2 p_{3 / 2} 3 s_{1 / 2}$ & 2 & $2 p_{3 / 2}^{2}$ & 0 & 9212.4 & $3.612 \times 10^{-6}$ & $2.6603 \times 10^{9}$ & 1.3460 \\
\hline $2 s_{1 / 2} 3 s_{1 / 2}$ & 1 & $2 s_{1 / 2} 2 p_{3 / 2}$ & 1 & 9240.8 & $5.824 \times 10^{-6}$ & $7.1928 \times 10^{9}$ & 1.3419 \\
\hline $2 p_{1 / 2} 3 s_{1 / 2}$ & 0 & $2 p_{1 / 2} 2 p_{3 / 2}$ & 2 & 9257.9 & $4.040 \times 10^{-6}$ & $1.5024 \times 10^{10}$ & 1.3394 \\
\hline $2 p_{1 / 2} 3 s_{1 / 2}$ & 1 & $2 p_{1 / 2} 2 p_{3 / 2}$ & 2 & 9260.0 & $6.055 \times 10^{-6}$ & $7.5093 \times 10^{9}$ & 1.3391 \\
\hline $2 p_{1 / 2} 3 s_{1 / 2}$ & 1 & $2 p_{1 / 2} 2 p_{3 / 2}$ & 1 & 9278.2 & $6.018 \times 10^{-6}$ & $7.4936 \times 10^{9}$ & 1.3365 \\
\hline $2 p_{3 / 2} 3 s_{1 / 2}$ & 2 & $2 p_{3 / 2}^{2}$ & 2 & 9281.5 & $1.514 \times 10^{-5}$ & $1.1316 \times 10^{10}$ & 1.3360 \\
\hline $2 p_{3 / 2} 3 s_{1 / 2}$ & 1 & $2 p_{3 / 2}^{2}$ & 2 & 9291.8 & $5.739 \times 10^{-6}$ & $7.1669 \times 10^{9}$ & 1.3345 \\
\hline $2 s_{1 / 2} 3 s_{1 / 2}$ & 1 & $2 s_{1 / 2} 2 p_{3 / 2}$ & 2 & 9331.8 & $6.256 \times 10^{-6}$ & $7.8799 \times 10^{9}$ & 1.3288 \\
\hline $2 s_{1 / 2} 3 s_{1 / 2}$ & 0 & $2 s_{1 / 2} 2 p_{3 / 2}$ & 2 & 9366.5 & $4.284 \times 10^{-6}$ & $1.6307 \times 10^{10}$ & 1.3239 \\
\hline $2 p_{3 / 2} 3 d_{5 / 2}$ & 2 & $2 p_{3 / 2}^{2}$ & 0 & 9872.0 & $1.030 \times 10^{-4}$ & $8.7096 \times 10^{10}$ & 1.2561 \\
\hline $2 p_{3 / 2} 4 s_{1 / 2}$ & 2 & $2 p_{3 / 2}^{2}$ & 0 & 12957.4 & $1.507 \times 10^{-6}$ & $2.1958 \times 10^{9}$ & 0.9570 \\
\hline $2 p_{1 / 2} 4 s_{1 / 2}$ & 1 & $2 p_{1 / 2} 2 p_{3 / 2}$ & 2 & 12995.5 & $2.076 \times 10^{-6}$ & $5.0722 \times 10^{9}$ & 0.9542 \\
\hline $2 s_{1 / 2} 4 s_{1 / 2}$ & 1 & $2 s_{1 / 2} 2 p_{3 / 2}$ & 1 & 12996.0 & $2.320 \times 10^{-6}$ & $5.6678 \times 10^{9}$ & 0.9541 \\
\hline $2 p_{1 / 2} 4 s_{1 / 2}$ & 0 & $2 p_{1 / 2} 2 p_{3 / 2}$ & 2 & 12999.9 & $1.635 \times 10^{-6}$ & $1.1992 \times 10^{10}$ & 0.9539 \\
\hline $2 p_{1 / 2} 4 s_{1 / 2}$ & 1 & $2 p_{1 / 2} 2 p_{3 / 2}$ & 1 & 13013.9 & $1.931 \times 10^{-6}$ & $4.7294 \times 10^{9}$ & 0.9528 \\
\hline $2 p_{3 / 2} 5 s_{1 / 2}$ & 2 & $2 p_{3 / 2}^{2}$ & 0 & 14655.3 & $7.596 \times 10^{-7}$ & $1.4159 \times 10^{9}$ & 0.8461 \\
\hline $2 p_{1 / 2} 4 d_{3 / 2}$ & 2 & $2 p_{1 / 2}^{2}$ & 0 & 14674.2 & $1.397 \times 10^{-6}$ & $2.6099 \times 10^{9}$ & 0.8450 \\
\hline $2 s_{1 / 2} 5 s_{1 / 2}$ & 1 & $2 s_{1 / 2} 2 p_{3 / 2}$ & 1 & 14696.5 & $1.149 \times 10^{-6}$ & $3.5911 \times 10^{9}$ & 0.8437 \\
\hline $2 p_{1 / 2} 5 s_{1 / 2}$ & 0 & $2 p_{1 / 2} 2 p_{3 / 2}$ & 2 & 14696.6 & $8.183 \times 10^{-7}$ & $7.6694 \times 10^{9}$ & 0.8437 \\
\hline $2 p_{1 / 2} 5 s_{1 / 2}$ & 1 & $2 p_{1 / 2} 2 p_{3 / 2}$ & 2 & 14697.3 & $1.203 \times 10^{-6}$ & $3.7584 \times 10^{9}$ & 0.8437 \\
\hline $2 p_{1 / 2} 6 s_{1 / 2}$ & 0 & $2 p_{1 / 2} 2 p_{3 / 2}$ & 2 & 15603.4 & $4.449 \times 10^{-7}$ & $4.7003 \times 10^{9}$ & 0.7947 \\
\hline $2 p_{1 / 2} 6 s_{1 / 2}$ & 1 & $2 p_{1 / 2} 2 p_{3 / 2}$ & 2 & 15603.9 & $6.544 \times 10^{-7}$ & $2.3045 \times 10^{9}$ & 0.7947 \\
\hline $2 s_{1 / 2} 6 s_{1 / 2}$ & 1 & $2 s_{1 / 2} 2 p_{3 / 2}$ & 1 & 15604.9 & $6.319 \times 10^{-7}$ & $2.2256 \times 10^{9}$ & 0.7946 \\
\hline $2 p_{1 / 2} 6 s_{1 / 2}$ & 1 & $2 p_{1 / 2} 2 p_{3 / 2}$ & 1 & 15622.3 & $6.617 \times 10^{-7}$ & $2.3358 \times 10^{9}$ & 0.7937 \\
\hline $2 p_{3 / 2} 6 s_{1 / 2}$ & 2 & $2 p_{3 / 2}^{2}$ & 2 & 15631.9 & $1.590 \times 10^{-6}$ & $3.3717 \times 10^{9}$ & 0.7932 \\
\hline $2 p_{3 / 2} 6 p_{3 / 2}$ & 3 & $2 s_{1 / 2} 2 p_{1 / 2}$ & 1 & 18902.9 & $2.028 \times 10^{-5}$ & $4.4924 \times 10^{10}$ & 0.6560 \\
\hline $2 p_{3 / 2} 6 p_{3 / 2}$ & 1 & $2 s_{1 / 2} 2 p_{1 / 2}$ & 1 & 18902.9 & $2.191 \times 10^{-5}$ & $1.1323 \times 10^{11}$ & 0.6560 \\
\hline $2 p_{3 / 2} 6 p_{3 / 2}$ & 2 & $2 s_{1 / 2} 2 p_{1 / 2}$ & 1 & 18905.1 & $2.739 \times 10^{-5}$ & $8.4954 \times 10^{10}$ & 0.6559 \\
\hline $2 p_{3 / 2} 6 p_{3 / 2}$ & 2 & $2 s_{1 / 2} 2 p_{1 / 2}$ & 0 & 18939.2 & $2.522 \times 10^{-5}$ & $7.8499 \times 10^{10}$ & 0.6547 \\
\hline
\end{tabular}


TABLE SV

Transition energy $(\Delta E[\mathrm{eV}])$, wavelength $(\lambda[\AA])$, radiative transition rates $\left(A_{r}\left[\mathrm{~s}^{-1}\right]\right)$ and weighted oscillator strengths $\left(g f_{i j}\right)$ for strong magnetic-octupole transitions (M3) of Be-like tungsten ion calculated by RCI method of FAC.

\begin{tabular}{|c|c|c|c|c|c|c|c|}
\hline Upper state & $J_{\text {up }}$ & Lower state & $J_{\text {low }}$ & $\Delta \mathrm{E}[\mathrm{eV}]$ & $g f_{i j}$ & $A_{r}\left[\mathrm{~s}^{-1}\right]$ & $\lambda[\AA]$ \\
\hline $2 p_{3 / 2} 3 p_{3 / 2}$ & 3 & $2 p_{3 / 2}^{2}$ & 0 & 9704.6 & $1.102 \times 10^{-7}$ & $6.4314 \times 10^{7}$ & 1.2777 \\
\hline $2 s_{1 / 2} 3 d_{5 / 2}$ & 2 & $2 p_{1 / 2} 2 p_{3 / 2}$ & 1 & 9746.0 & $1.571 \times 10^{-8}$ & $1.2953 \times 10^{7}$ & 1.2723 \\
\hline $2 s_{1 / 2} 3 p_{3 / 2}$ & 2 & $2 s_{1 / 2} 2 p_{3 / 2}$ & 1 & 9758.5 & $1.637 \times 10^{-7}$ & $1.3526 \times 10^{8}$ & 1.2707 \\
\hline $2 p_{1 / 2} 3 p_{3 / 2}$ & 1 & $2 p_{1 / 2} 2 p_{3 / 2}$ & 2 & 9767.3 & $1.688 \times 10^{-7}$ & $2.3298 \times 10^{8}$ & 1.2695 \\
\hline $2 p_{1 / 2} 3 p_{3 / 2}$ & 2 & $2 p_{1 / 2} 2 p_{3 / 2}$ & 2 & 9772.2 & $1.009 \times 10^{-7}$ & $8.3600 \times 10^{7}$ & 1.2689 \\
\hline $2 p_{3 / 2} 3 p_{3 / 2}$ & 3 & $2 p_{3 / 2}^{2}$ & 2 & 9773.7 & $2.687 \times 10^{-7}$ & $1.5912 \times 10^{8}$ & 1.2687 \\
\hline $2 p_{3 / 2} 3 p_{3 / 2}$ & 1 & $2 p_{3 / 2}^{2}$ & 2 & 9774.7 & $6.719 \times 10^{-8}$ & $9.2852 \times 10^{7}$ & 1.2686 \\
\hline $2 p_{1 / 2} 3 p_{3 / 2}$ & 2 & $2 p_{1 / 2} 2 p_{3 / 2}$ & 1 & 9790.4 & $1.543 \times 10^{-7}$ & $1.2838 \times 10^{8}$ & 1.2665 \\
\hline $2 p_{3 / 2} 3 p_{3 / 2}$ & 2 & $2 p_{3 / 2}^{2}$ & 2 & 9806.6 & $2.277 \times 10^{-7}$ & $1.9006 \times 10^{8}$ & 1.2645 \\
\hline $2 s_{1 / 2} 3 p_{3 / 2}$ & 2 & $2 s_{1 / 2} 2 p_{3 / 2}$ & 2 & 9849.5 & $1.183 \times 10^{-7}$ & $9.9574 \times 10^{7}$ & 1.2589 \\
\hline $2 p_{3 / 2} 4 p_{3 / 2}$ & 3 & $2 p_{3 / 2}^{2}$ & 0 & 13164.0 & $5.284 \times 10^{-8}$ & $5.6765 \times 10^{7}$ & 0.9420 \\
\hline $2 s_{1 / 2} 4 p_{3 / 2}$ & 2 & $2 s_{1 / 2} 2 p_{3 / 2}$ & 1 & 13210.4 & $7.454 \times 10^{-8}$ & $1.1289 \times 10^{8}$ & 0.9387 \\
\hline $2 p_{1 / 2} 4 p_{3 / 2}$ & 1 & $2 p_{1 / 2} 2 p_{3 / 2}$ & 2 & 13211.8 & $8.053 \times 10^{-8}$ & $2.0331 \times 10^{8}$ & 0.9386 \\
\hline $2 p_{1 / 2} 4 p_{3 / 2}$ & 2 & $2 p_{1 / 2} 2 p_{3 / 2}$ & 2 & 13212.2 & $5.338 \times 10^{-8}$ & $8.0872 \times 10^{7}$ & 0.9385 \\
\hline $2 p_{1 / 2} 4 p_{3 / 2}$ & 2 & $2 p_{1 / 2} 2 p_{3 / 2}$ & 1 & 13230.7 & $8.108 \times 10^{-8}$ & $1.2317 \times 10^{8}$ & 0.9372 \\
\hline $2 p_{1 / 2} 4 f_{5 / 2}$ & 3 & $2 p_{1 / 2}^{2}$ & 0 & 14734.6 & $1.129 \times 10^{-8}$ & $1.5198 \times 10^{7}$ & 0.8416 \\
\hline $2 p_{1 / 2} 4 f_{7 / 2}$ & 3 & $2 p_{1 / 2}^{2}$ & 0 & 14759.8 & $3.100 \times 10^{-7}$ & $4.1869 \times 10^{8}$ & 0.8401 \\
\hline $2 p_{3 / 2} 5 p_{3 / 2}$ & 3 & $2 p_{3 / 2}^{2}$ & 0 & 14760.2 & $3.345 \times 10^{-8}$ & $4.5172 \times 10^{7}$ & 0.8401 \\
\hline $2 p_{3 / 2} 4 f_{5 / 2}$ & 3 & $2 p_{1 / 2} 2 p_{3 / 2}$ & 2 & 14782.1 & $7.413 \times 10^{-9}$ & $1.0041 \times 10^{7}$ & 0.8389 \\
\hline $2 p_{3 / 2} 4 f_{5 / 2}$ & 2 & $2 p_{1 / 2} 2 p_{3 / 2}$ & 2 & 14784.5 & $8.076 \times 10^{-9}$ & $1.5319 \times 10^{7}$ & 0.8387 \\
\hline $2 p_{3 / 2} 6 p_{3 / 2}$ & 3 & $2 p_{3 / 2}^{2}$ & 0 & 15623.9 & $2.132 \times 10^{-8}$ & $3.2262 \times 10^{7}$ & 0.7937 \\
\hline $2 p_{3 / 2} 6 f_{7 / 2}$ & 3 & $2 p_{3 / 2}^{2}$ & 0 & 15653.7 & $1.960 \times 10^{-7}$ & $2.9771 \times 10^{8}$ & 0.7921 \\
\hline $2 p_{1 / 2} 6 p_{3 / 2}$ & 1 & $2 p_{1 / 2} 2 p_{3 / 2}$ & 2 & 15665.8 & $3.241 \times 10^{-8}$ & $1.1505 \times 10^{8}$ & 0.7915 \\
\hline $2 p_{1 / 2} 6 p_{3 / 2}$ & 2 & $2 p_{1 / 2} 2 p_{3 / 2}$ & 2 & 15666.1 & $2.166 \times 10^{-8}$ & $4.6132 \times 10^{7}$ & 0.7915 \\
\hline $2 s_{1 / 2} 6 p_{3 / 2}$ & 2 & $2 s_{1 / 2} 2 p_{3 / 2}$ & 1 & 15667.9 & $2.648 \times 10^{-8}$ & $5.6411 \times 10^{7}$ & 0.7914 \\
\hline
\end{tabular}

